\title{
Cifoplastia guiada por TC: Una opción técnica en el tratamiento de fracturas vertebrales patológicas
}

Dres. Amadeo Muntané $\mathbf{S}^{(1)}$, Daniel Rodríguez $B^{(1)}$, Víctor Mayoral $\mathbf{R}^{(2)}$, Paloma Mora $M^{(1)}$, Lucía Aja $\boldsymbol{R}^{(1)}$, Sonia Aixut $L^{(1)}$.

1. Servicio de Radiodiagnóstico. Departamento de Neurorradiología, Hospital Universitario de Bellvitge. L'Hospitalet (Barcelona), España.

2. Servicio de Anestesiología, Clínica del Dolor. L’Hospitalet (Barcelona), España.

\section{CT-guided kyphoplasty: A technical option in the treatment of pathological vertebral fractures}

\begin{abstract}
Pathological vertebral fractures are caused by various entities. They cause significant pain and impaired quality of life of patients. The CT-guided kyphoplasty relieves or eliminates pain and stabilizes the fractured vertebral bodies. 49 patients were treated. The procedure is performed percutaneously by inserting a needle that is subsequently removed, leaving a cannula. Through this a balloon-like device is introduced and subsequently inflated to create a cavity, which is then filled with polymethylmethacrylate (PMMA). The technique was performed successfully in all cases without serious complications, with good results. Its advantages are that the needle placement as well as the injection of PMMA can be correctly visualized using real-time CT fluoroscopy. In addition a single needle is used throughout the entire procedure, which minimizes the risk of complications as it is less traumatic.
\end{abstract}

Keywords: CT scan, Kyphoplasty, Pain, Polymethylmethacrylate, Vertebral fracture.

Resumen. Las fracturas vertebrales patológicas se originan por diversas entidades. Producen dolor importante y deterioro de la calidad de vida de los pacientes. La cifoplastia guiada por tomografía computarizada (TC) alivia o elimina el dolor y estabiliza los cuerpos vertebrales fracturados. Se han tratado 49 pacientes. El procedimiento se realiza por vía percutánea, mediante la inserción de una aguja que posteriormente se retira dejando una cánula. A través de la misma se introduce un dispositivo que lleva incorporado un balón que se infla creando una cavidad, que se rellena con polimetilmetacrilato (PMMA). La técnica se practicó con éxito en todos los casos sin complicaciones graves, con buenos resultados. Sus ventajas son visualizar correctamente la posición de la aguja y la inyección del PMMA mediante la escopia del TC en tiempo real. Además se utiliza una sola aguja para todo el procedimiento, lo cual minimiza el riesgo de complicaciones siendo menos traumático.

Palabras clave: Cifoplastia, Dolor, Fractura vertebral, Polimetilmetacrilato, Tomografía Computarizada.

Muntané A. Cifoplastia guiada por TC: Una opción técnica en el tratamiento de fracturas vertebrales patológicas. Rev Radiol 2013; 19(4): 150-155.

Correspondencia: Amadeo Muntané / amuntane@bellvitgehospital.cat

Trabajo recibido el 26 de abril de 2013. Aceptado el 18 de diciembre de 2013.

\section{Introducción}

La etiología de las fracturas vertebrales patológicas es diversa. La causa más común es la osteoporosis; sin embargo, las metástasis vertebrales y el mieloma también pueden ocasionar una fractura vertebral que requiera tratamiento. La cifoplastia es un procedimiento intervencionista en el cual, por vía percutánea, se introduce una aguja con una cánula en el cuerpo vertebral lesionado. Posteriormente se retira la aguja y se introduce por la cánula un balón que se infla dando lugar a una cavidad en el interior del cuerpo vertebral. Seguidamente se inyecta cemento en la cavidad creada por el balón. Los objetivos básicos de la realización de la cifoplastia son el alivio del dolor y la estabilización de la vértebra. La exposición de esta serie de casos obedece a poner de relieve esta técnica como una opción más en el tratamiento de las fracturas vertebrales patológicas y además mostrar su valor en la mejora de la sintomatología clínica ${ }^{(1,2)}$.

\section{Material y métodos}

Se ha tratado a 49 pacientes (18 hombres y 31 mujeres) con un número total de 100 fracturas vertebrales patológicas desde enero de 2010 hasta mayo de 2012. Las edades de los pacientes oscilaron entre 43 y 88 años (edad media 71,5 años). Se incluyeron todos los pacientes que presentaban dolor en la columna vertebral con sospecha clínica de fractura 
aguda confirmada mediante resonancia magnética. La osteoporosis fue la causa principal de esta serie de casos, mientras que el resto fue de causa tumoral (Tabla I). Se hubieran excluido los pacientes afectos de espondilodiscitis, sepsis, coagulopatía y patología cardiopulmonar severa que contraindicara el procedimiento. Ninguno de los pacientes presentó estos criterios de exclusión. No se consideró criterio de exclusión el hecho de que se apreciara afectación del muro posterior en una vértebra fracturada a tratar. En ningún paciente se identificó compresión medular. El dolor se midió con la escala visual analógica (EVA). Esta escala fue empleada inicialmente por Huskinson en 1976. Consiste en dibujar una línea, limitada en sus dos extremos por dos líneas perpendiculares, la porción inferior/izquierda representa ningún dolor y la parte superior/derecha el máximo dolor experimentado. Esta escala es considerada la mejor porque es

Tabla I. En esta tabla puede observarse el número de paciente, sexo, edad, la etiología de la fractura, el EVA antes y después del procedimiento, los ml de PMMA inyectados en cada vértebra y las vértebras afectadas en cada paciente.

\begin{tabular}{|c|c|c|c|c|c|c|c|}
\hline$N^{\circ}$ Paciente & Sexo & Edad & Etiología & $\begin{array}{l}\text { EVA } \\
\text { antes }\end{array}$ & $\begin{array}{c}\text { EVA } \\
\text { después }\end{array}$ & $\begin{array}{l}\text { mI de PMMA inyectadas } \\
\text { en cada vertebra }\end{array}$ & $\begin{array}{l}\text { Vertebras } \\
\text { afectadas }\end{array}$ \\
\hline 1 & $\mathrm{H}$ & 78 & Metástasis & 9 & 2 & 1 & D12-L3 \\
\hline 2 & M & 80 & Mieloma & 8 & 3 & 1 & L3 \\
\hline 3 & M & 77 & Osteoporosis & 8 & 4 & 1 & D12-L1-L2 \\
\hline 4 & $M$ & 57 & Metástasis & 8 & 3 & 1 & D7-D8 \\
\hline 5 & $M$ & 60 & Metástasis & 9 & 2 & 2 & D8 \\
\hline 6 & M & 65 & Metástasis & 10 & 5 & 1 & D12-L2-L3-L4-L5 \\
\hline 7 & $\mathrm{H}$ & 73 & Osteoporosis & 10 & 4 & 1 & D8-D9- \\
\hline 8 & $\mathrm{H}$ & 65 & Mieloma & 9 & 2 & 1 & D4-D10-D12-L2 \\
\hline 9 & $\mathrm{H}$ & 75 & Osteoporosis & 8 & 3 & 1 & D10-L1 \\
\hline 10 & $\mathrm{H}$ & 82 & Osteoporosis & 8 & 3 & 1 & L1-L4 \\
\hline 11 & M & 82 & Osteoporosis & 9 & 3 & 2 & L2 \\
\hline 12 & $\mathrm{H}$ & 79 & Osteoporosis & 9 & 2 & 1 & D11-D12-L2-L4 \\
\hline 13 & M & 65 & Mieloma & 8 & 2 & 1 & D1-D7-D8-L1 \\
\hline 14 & $\mathrm{M}$ & 86 & Osteoporosis & 8 & 2 & 2 & L5 \\
\hline 15 & M & 78 & Osteoporosis & 10 & 1 & 2 & D12 \\
\hline 16 & $\mathrm{H}$ & 72 & Osteoporosis & 10 & 3 & 1 & D12-L1-L2-L5 \\
\hline 17 & M & 71 & Osteoporosis & 10 & 4 & 1 & D7-D8-D11-L1-L3-L4 \\
\hline 18 & $\mathrm{H}$ & 78 & Metástasis & 8 & 3 & 1 & D5-D8-L1 \\
\hline 19 & M & 76 & Osteoporosis & 8 & 3 & 2 & L1 \\
\hline 20 & M & 80 & Osteoporosis & 9 & 8 & 2 & L4 \\
\hline 21 & M & 77 & Osteoporosis & 8 & 3 & 2 & L1 \\
\hline 22 & M & 73 & Osteoporosis & 8 & 4 & 1 & D9-D12 \\
\hline 23 & M & 72 & Osteoporosis & 9 & 3 & 1 & L3 \\
\hline 24 & M & 74 & Metástasis & 8 & 3 & 2 & L1 \\
\hline 25 & $\mathrm{H}$ & 72 & Osteoporosis & 7 & 2 & 1 & L1-L2 \\
\hline 26 & M & 67 & Osteoporosis & 9 & 0 & 2 & L3 \\
\hline 27 & $\mathrm{H}$ & 63 & Osteoporosis & 10 & 3 & 1 & D7-L1-L4-L5 \\
\hline 28 & $\mathrm{H}$ & 43 & Metástasis & 8 & 7 & 2 & L3 \\
\hline 29 & M & 63 & Mieloma & 8 & 1 & 2 & L2 \\
\hline 30 & $\mathrm{H}$ & 54 & Metástasis & 10 & 2 & 2 & L2 \\
\hline 31 & M & 85 & Osteoporosis & 8 & 2 & 2 & L2 \\
\hline 32 & M & 85 & Osteoporosis & 7 & 2 & 2 & L1 \\
\hline 33 & M & 56 & Mieloma & 9 & 2 & 2 & S2 \\
\hline 34 & $\mathrm{H}$ & 73 & Metástasis & 9 & 8 & 1 & L1-L2-L3 \\
\hline 35 & M & 79 & Osteoporosis & 8 & 3 & 1 & D9-D12-L1 \\
\hline 36 & M & 64 & Osteoporosis & 8 & 2 & 1 & D12-L1 \\
\hline 37 & $\mathrm{H}$ & 52 & Metástasis & 10 & 1 & 1 & D12-L3-L4-L5-S1 \\
\hline 38 & $\mathrm{H}$ & 70 & Mieloma & 10 & 4 & 1 & D7 \\
\hline 39 & M & 86 & Osteoporosis & 9 & 3 & 2 & D11 \\
\hline 40 & $\mathrm{H}$ & 56 & Osteoporosis & 9 & 3 & 1 & D6 \\
\hline 41 & $\mathrm{H}$ & 72 & Metástasis & 8 & 3 & 1 & D2 \\
\hline 42 & M & 78 & Osteoporosis & 10 & 4 & 2 & $\mathrm{~L} 1$ \\
\hline 43 & M & 69 & Osteoporosis & 9 & 3 & 1 & L1-L2-L3-L4 \\
\hline 44 & M & 70 & Osteoporosis & 8 & 2 & 2 & D12 \\
\hline 45 & M & 72 & Osteoporosis & 8 & 2 & 2 & L3 \\
\hline 46 & $\mathrm{H}$ & 72 & Osteoporosis & 9 & 3 & 2 & L1 \\
\hline 47 & M & 68 & Osteoporosis & 8 & 2 & 2 & L1 \\
\hline 48 & $\mathrm{H}$ & 82 & Osteoporosis & 8 & 4 & 1 & L1-L3 \\
\hline 49 & M & 73 & Osteoporosis & 9 & 1 & 1 & L1-L3 \\
\hline \multicolumn{4}{|c|}{ Número Total de pacientes } & 49 & & & \\
\hline \multicolumn{4}{|c|}{ Número Total vértebras } & 100 & & & \\
\hline
\end{tabular}


práctica y fiable, a la vez que su realización no es complicada ${ }^{(3)}$. Hemos dado el cero como valor numérico equivalente a ausencia de dolor y el número diez como el máximo dolor experimentado.

Los pacientes fueron derivados desde la clínica del dolor, oncología, hematología y medicina interna. Por otro lado, se les realizaron pruebas de laboratorio, radiografía de tórax, electrocardiograma y valoración anestésica. A todos los pacientes se les realizó Resonancia Magnética de la columna, en donde las vértebras afectadas mostraron baja intensidad de señal en imágenes potenciadas en T1 y alta intensidad de señal en secuencia STIR. Todos ellos firmaron el consentimiento informado.

Para la realización de esta técnica se ha utilizado un TC con fluoroscopia en tiempo real (Lightspeed 16; General Electric, Milwaukee, WI, EE.UU.), el cual proporciona una visión excelente del posicionamiento de las agujas, así como de la distribución del cemento dentro del cuerpo vertebral. El procedimiento debe llevarse a cabo en condiciones de asepsia adecuadas en la sala de TC. El paciente se coloca en posición prono y recibe una sedación leve por parte del anestesiólogo, que permanece en la sala durante la realización de la cifoplastia. Posteriormente, es necesario determinar el punto de entrada de la aguja en la piel, así como la dirección y la profundidad de la misma. Para ello, se realiza una TC con las reconstrucciones y mediciones pertinentes. Se ha efectuado un único acceso a la vértebra. En las vértebras dorsales ha sido la vía costotransversa; en las lumbares el acceso posterolateral, y en los segmentos sacros S1 y S2 de dos pacientes respectivamente, se ha decidido el acceso directo evitando los agujeros sacros adyacentes. Estas vías de abordaje han sido las más apropiadas en nuestros casos. No obstante se puede utilizar también la vía transpedicular y yuxtapedicular, dado que el control de imagen por TC lo permite con cierta facilidad. Sin embargo, no se han usado porque en la mayoría de los casos hubiera sido necesario introducir dos agujas.

Por otra parte, han sido mucho más cómodos los accesos anteriormente mencionados para colocar las agujas de entrada en la situación correcta y pertinente. Se puede realizar un topograma para poder determinar la dirección craneocaudal de la aguja (Figura 1). Una vez definido el punto de entrada se procede a anestesiar la piel y a realizar una pequeña incisión en la misma para introducir la aguja canulada para cifoplastia (Kyphon Express Medtronic). Después de colocar la aguja se retira dejando la cánula (Figura 2). A través de la cánula se introduce una broca para crear un espacio en el interior del cuerpo vertebral (Figura 3). Retirada la broca se introduce un balón, que tiene dos marcadores radiopacos para identificarlo y de este modo realizar una colocación precisa (Figura 4). Este balón se infla mediante un sistema de inyección de contraste que mide la presión mediante un manómetro digital (Figura 5). Se han utilizado dos tamaños de balones, de 10 y $15 \mathrm{~mm}$ de longitud, con un volumen de 4 a $5 \mathrm{ml}$, respectivamente, ambos con una presión máxima de inflado de $400 \mathrm{PSI}$. EI balón tiene la misión de configurar una cavidad en el cuerpo vertebral por la compresión de las trabéculas adyacentes. El tiempo que permanecieron inflados los balones fue de 4-5-minutos. Posteriormente se procedió al desinflado del balón y a su retirada. Luego, mediante un dispositivo que se introduce también a través de la cánula, el PMMA es inyectado a baja presión con una cantidad entre 1 y $2 \mathrm{ml}$ por segmento vertebral (Tabla I), rellenando la cavidad confeccionada por el balón (Figuras 6, 7, 8, 9 y 10). La TC con fluoroscopia en tiempo real permite una excelente visualización de todo el proceso, tanto en el plano axial como el eje craneocaudal, ya que la mesa se mueve en esta última dirección.

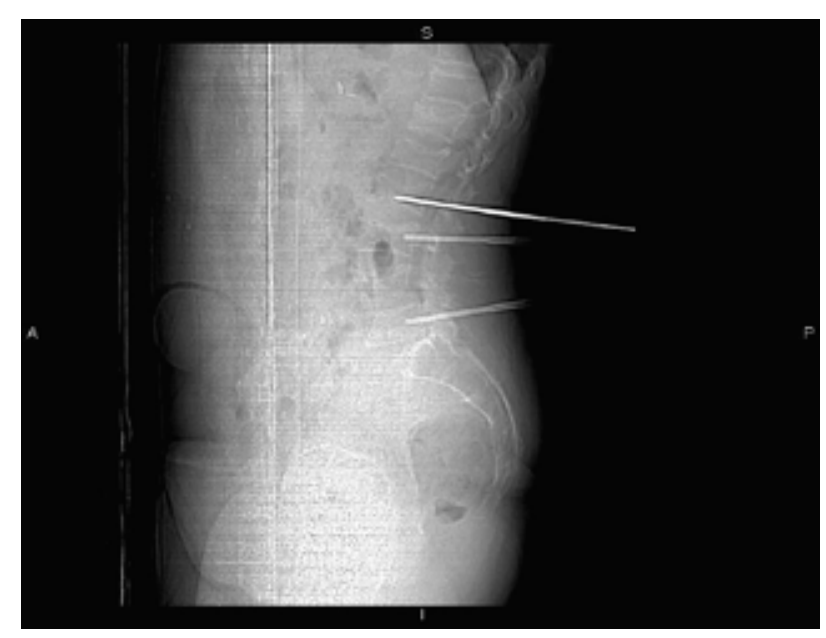

Figura 1. Realización de topograma para determinar la dirección de las agujas.

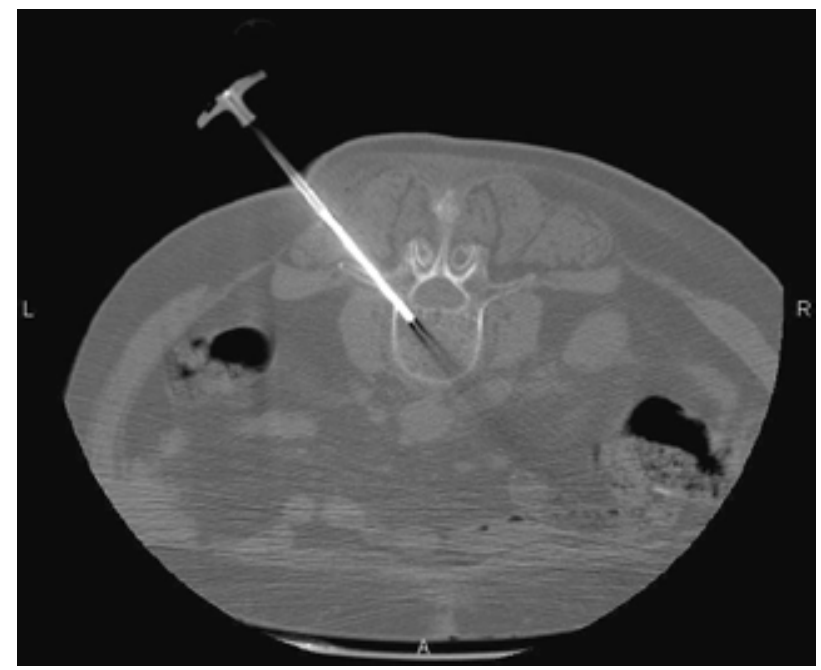

Figura 2. TC corte axial. Se puede observar la colocación de la cánula. 


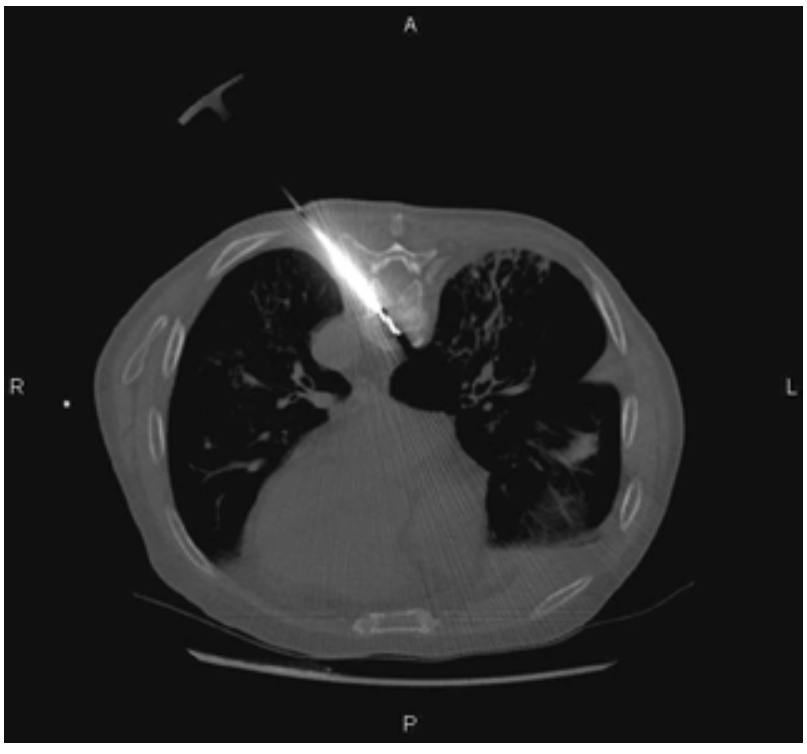

Figura 3. TC corte axial. Colocación de la broca (flecha).

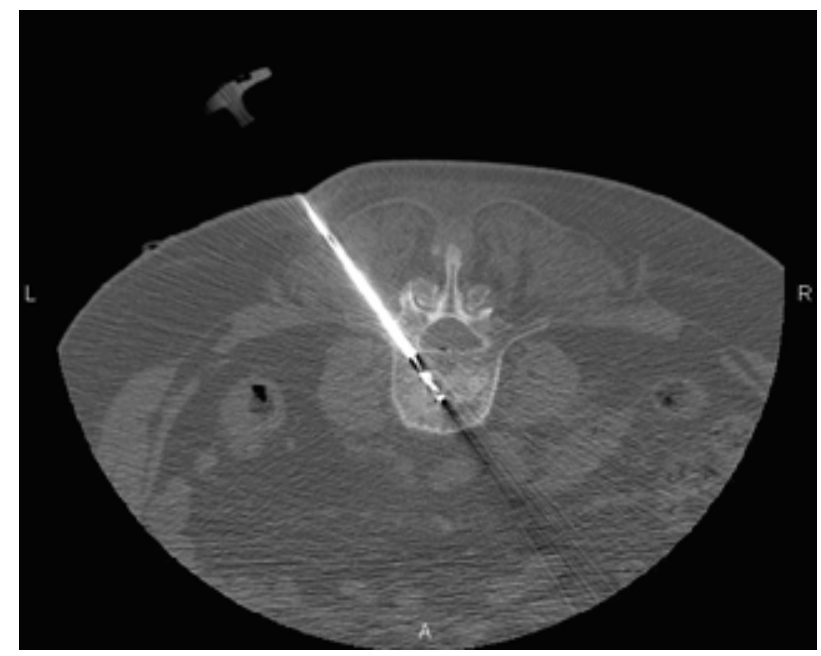

Figura 4. Introducción del balón desinflado dentro del cuerpo vertebral.

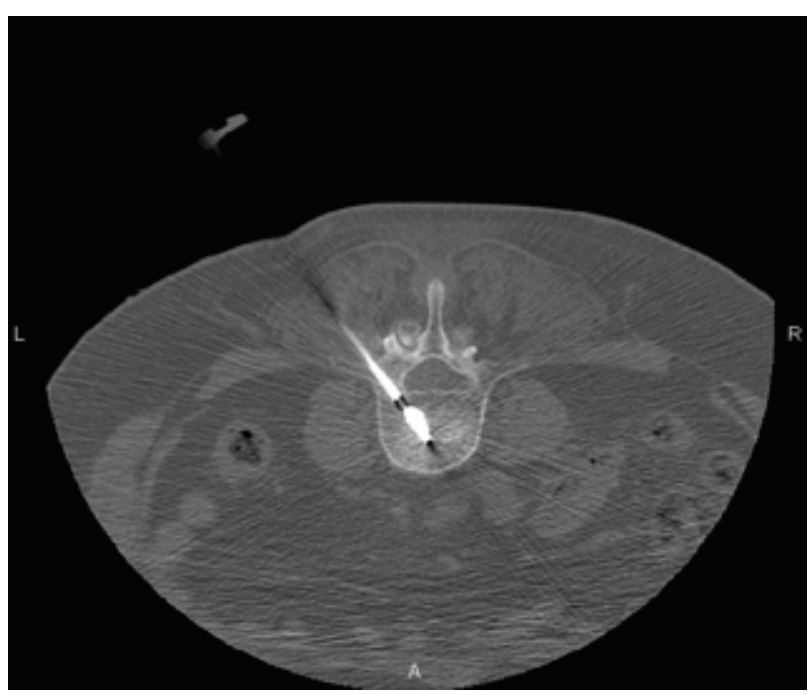

Figura 5. Balón inflado en el interior del cuerpo vertebral.

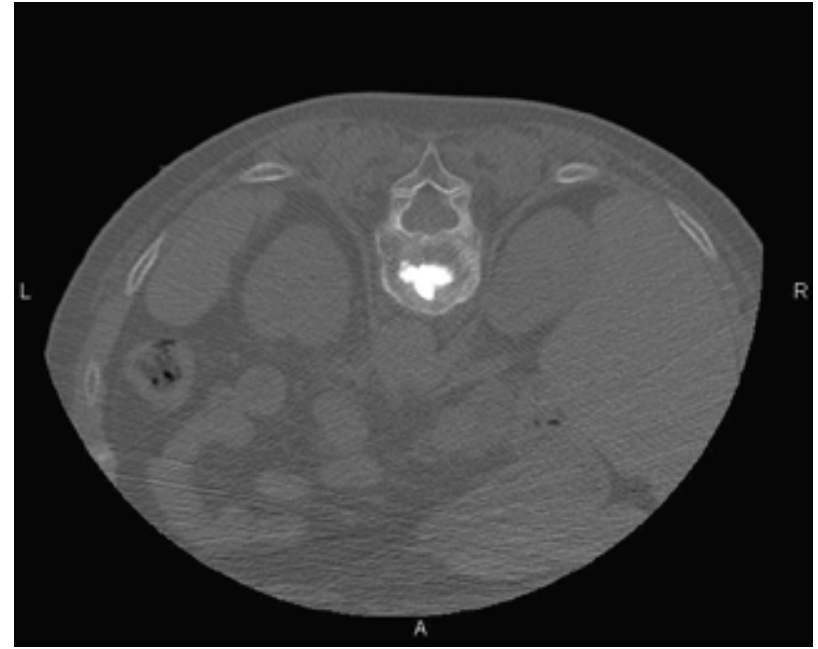

Figura 6. Inyección de PMMA.

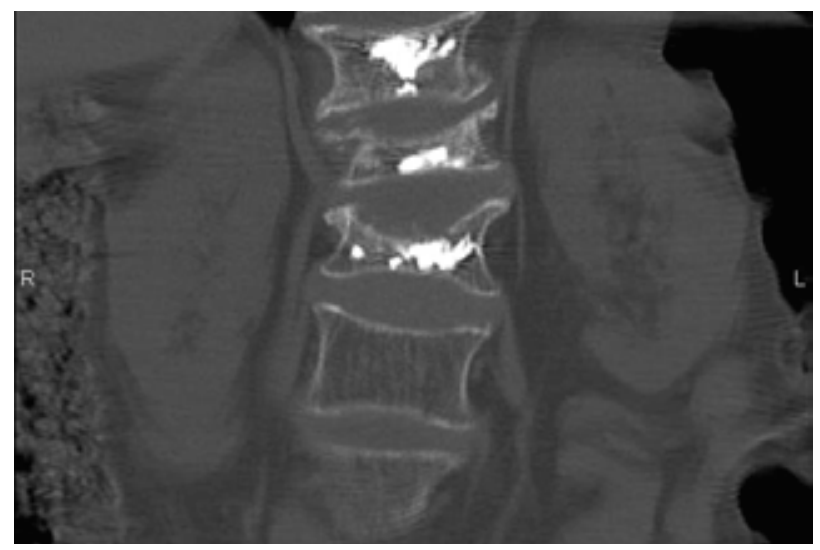

Figura 7. TC reconstrucción coronal. Se observa el cemento inyectado en los cuerpos vertebrales D12, L1 y L2.

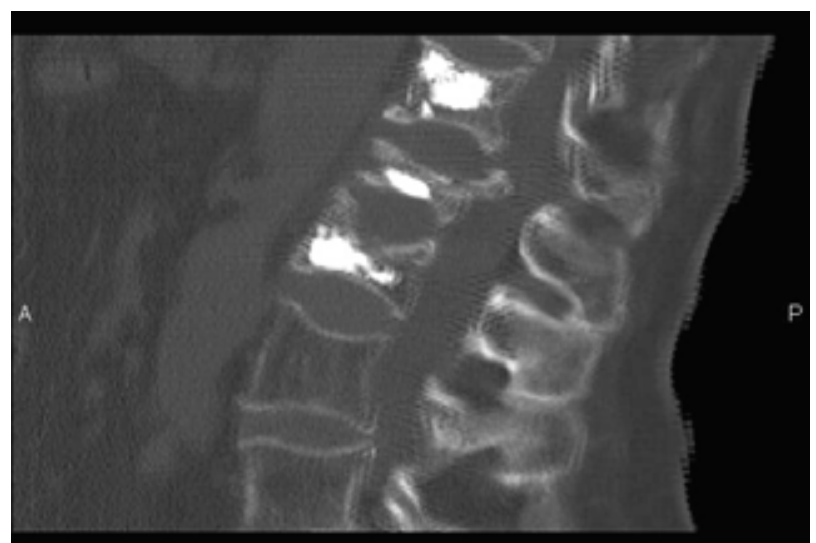

Figura 8. TC reconstrucción sagital de las vértebras D12, L1 y L2 tratadas. 


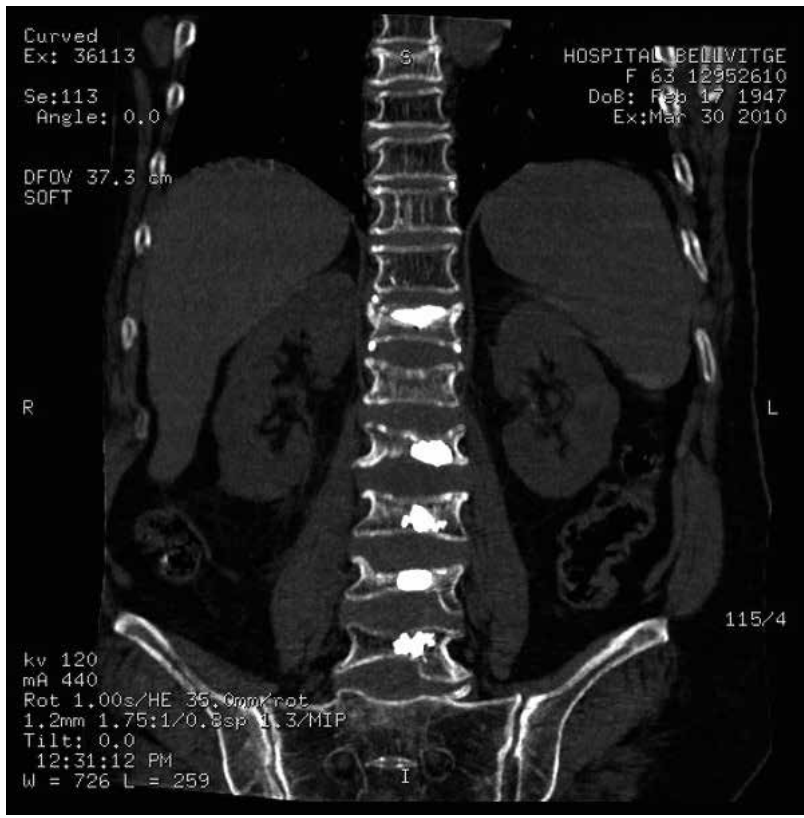

Figura 9. TC reconstrucción coronal. Cifoplastia en D12, L2, L3, L4 y L5.

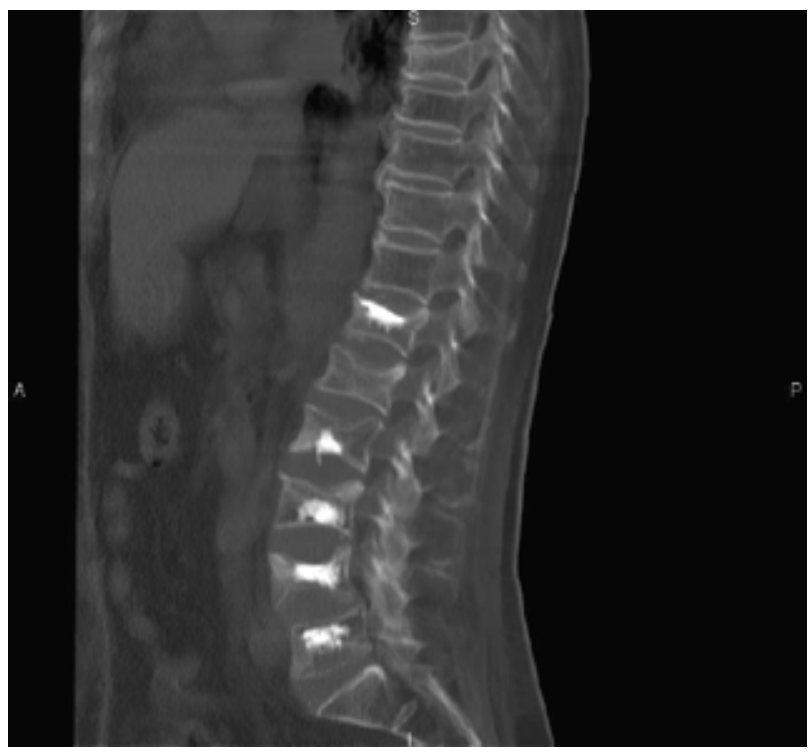

Figura 10. TC reconstrucción sagital de la cifoplastia múltiple de D12, L2, L3, L4 y L5.

\section{Resultados}

En todos los casos el cemento se inyectó dentro del cuerpo vertebral con una distribución correcta. El período de seguimiento se estableció desde la intervención hasta 24 meses después. El 90\% de los pacientes mostró una mejoría significativa en el dolor inmediatamente después del procedimiento. El 4\% la experimentó 24-36 horas post-procedimiento. El 6\%, que representan tres pacientes, no mejoró de manera franca la intensidad del dolor, lo cual desconocemos a qué atribuirlo. No hubo diferencias significativas en cuanto a la disminución del EVA en relación a las diferentes vértebras tratadas (Tabla I).

Las complicaciones derivadas del procedimiento fueron mínimas. En sólo tres casos se detectó una extravasación de PMMA local sin consecuencias clínicas. En dos pacientes que se medicaban con anticoagulantes y que la analítica permitió la realización del procedimiento, desarrollaron un hematoma en el músculo psoas relacionado con la punción, pero en ningún caso hubo una repercusión clínica significativa.

\section{Discusión}

La cifoplastia es una técnica óptima para el tratamiento de fracturas patológicas. La mejoría rápida, conspicua y sostenida del dolor, así como la recuperación funcional de los pacientes después del procedimiento, hacen que la cifoplastia sea una técnica válida para mejorar la calidad de vida de estos enfermos ${ }^{(2,4-7)}$.

Habitualmente los pacientes con fracturas vertebrales tratadas con vertebroplastia o cifoplastia, se han realizado con fluoroscopia con un arco en $\mathrm{C}$ de rayos $\mathrm{X}$ o con un angiógrafo. Se ha descrito la combinación de TC y fluoroscopia utilizando un arco en $\mathrm{C}$ de rayos $\mathrm{X}$ incorporado en el $\mathrm{TC}^{(8)}$. Con estos dispositivos radiológicos el acceso de las agujas hacia el interior del cuerpo vertebral se realiza normalmente por vía transpedicular bilateral. En nuestra serie de pacientes hemos utilizado el TC con fluoroscopia en tiempo real, de manera que pueden obtenerse imágenes axiales tomográficas en el mismo momento que se realiza escopia. Por otra parte, utilizando esta técnica no es necesario acceder al cuerpo vertebral patológico a través de los pedículos bilateralmente, sino que es posible utilizar una única vía de entrada. Se utiliza una sola aguja por segmento vertebral, por lo tanto, esta forma de realizar este intervencionismo es menos agresiva. También hay que considerar que al utilizar la TC con fluoroscopia en tiempo real, se pone de relieve perfectamente la distribución de cemento y en caso de que exista fuga hacia una vena paravertebral se identifica con precisión y se puede parar la inyección de PMMA.

Hay que tener en cuenta que la vertebroplastia es igualmente eficaz en el tratamiento del dolor incapacitante producido por fracturas vertebrales patológicas $^{(9,10)}$. Sin embargo, la extravasación del cemento en el sistema venoso vertebral y en los discos intervertebrales es frecuente. No obstante la mayoría de los autores han informado que dicha extravasación se presenta de forma asintomática en la mayor parte de los casos ${ }^{(8,11,12)}$. En la cifoplastia es posible que haya una fuga de cemento, pero la frecuencia es menor. Sólo hubo tres casos de extravasación discal en 49 vértebras tratadas, considerando además que en un paciente se realizó tratamiento en seis niveles en una sola sesión. Mediante la 
vertebroplastia también se han tratado varios niveles vertebrales con eficacia óptima para mejorar el dolor ${ }^{(13)}$. No obstante, se ha descrito una muerte por embolia pulmonar grasa al realizar vertebroplastia en tres niveles: D10, D11 y D12. La embolia grasa se caracteriza por la liberación de partículas de grasa en la circulación sistémica después de un trauma. Una vez que ha producido una fractura, la grasa se libera en la circulación venosa y puede obstruir los capilares. En este caso, los autores propusieron la hipótesis de que al introducir el PMMA en el cuerpo vertebral, provoca un aumento en la presión intramedular. Esta presión provoca un desplazamiento de la grasa de la médula ósea en las estructuras de menor resistencia, tales como la circulación venosa. El volumen de PMMA que utilizaron fue de $5 \mathrm{ml}$ en $\mathrm{D} 10,6 \mathrm{ml}$ en $\mathrm{D} 11$ y $5 \mathrm{ml}$ en $\mathrm{D} 12^{(14)}$.

Hay que decir que no se ha encontrado una asociación significativa entre el volumen de cemento inyectado y los resultados clínicos en relación con el dolor después del procedimiento. Además, grandes volúmenes de PMMA inyectado dan lugar a un mayor riesgo de complicaciones relacionadas con la fuga de cemento ${ }^{(15)}$. Por esta razón, con el fin de evitar complicaciones graves, se recomienda reducir el volumen de cemento inyectado. En nuestros pacientes generalmente se inyecta de 1-2 $\mathrm{ml}$ de cemento en un cuerpo vertebral fracturado. A nuestro juicio con la cifoplastia, el inflado del balón dentro del cuerpo vertebral produce una compresión de las trabéculas óseas y al mismo tiempo un colapso de las venas somáticas, lo cual da lugar a una cavidad en la que posteriormente se deposita el cemento al inyectarlo. Este hecho y el poco volumen de cemento que se administra evita las fugas y las extravasaciones de una forma más eficiente que en el caso de la vertebroplastia.

Cabe mencionar que un reciente estudio ha evaluado el riesgo relativo de mortalidad en pacientes con fractura vertebral que fueron tratadas con vertebroplastia, este riesgo es de un $23 \%$ mayor que en los pacientes que fueron tratados con cifoplastia ${ }^{(16)}$.

\section{Conclusión}

La cifoplastia guiada por TC es una opción técnica adecuada en el tratamiento de las fracturas vertebrales patológicas. El hecho de acceder a la vértebra afectada por una única entrada hace que el procedimiento sea menos traumático y por consiguiente con menos riesgo de complicaciones.

\section{Bibliografía}

1. Cloft HJ, Jensen ME. Kyphoplasty: An Assessment of a New Technology AJNR Am J Neuroradiol 2007; 28: 200-203.

2. Garfin SR, Buckley RA, Ledlie J. Balloon kyphoplasty for symptomatic vertebral body compression fractures results in rapid, significant and sustained improvements in back pain, function, and quality of life for elderly pacients. Spine 2006; 31(19): 2213-2220.

3. Melzack R. The McGill painquestionaire: Major properties and scoring methods in pain. Pain 1975; 1: 277-99.

4. Ledlie JT, Renfro MB. Kyphoplasty treatment of vertebral fractures: 2-year outcomes show sustained benefits. Spine 2006; 31(1): 57-64.

5. Center JR, Nguyen TV, Schneider D, et al. Mortality after major types of osteoporotic fracture in men and woman: An observational study. Lancet 1999; 353: 878-882.

6. Cooper C, Atkinson EJ, Jacobsen SJ, et al. Populationbased study of survival after osteoporotic fractures. Am J Epidemiol 1993; 137: 1001-1005.

7. Schlaich C, Minne HW, Bruckner T, et al. Reduced pulmonary function in patients with spinal osteoporotic fractures. OsteoporosInt 1998; 8: 261-267.

8. Silverman SL. The clinical consequences of vertebral compression fracture. Bone 1992; 13 Suppl 2: 27-31.

9. Deramond H, Saliou G, Aveillan M, et al. Respective contributions of vertebroplasty and kyphoplasty to the management of osteoporotic vertebral fractures. Joint Bone Spine 2006; 73(6): 610-613.

10. Evans AJ, Jensen ME, Kip KE, et al. Vertebral compression fractures: Pain reduction and improvement in functional mobility after percutaneous polymethylmethacrylatevertebroplasty retrospective report of 245 cases. Radiology 2003; 226(2): 366-372.

11. Baumann $\mathrm{C}$, Fuchs $\mathrm{H}$, Kiwit J, et al. Complications in percutaneous vertebroplasty associated with puncture or cement leakage. Cardiovasc Intervent Radiol 2007; 30(2): 161-168.

12. Barragan-Campos HM, Vallée JN, Lo D, et al. Percutaneous vertebroplasty for spinal metastases: Complications. Radiology 2006; 238(1): 354-362.

13. Singh AK, Pilgram TK, Gilula LA. Osteoporotic compression fractures: Outcomes after single-versus multiple-level percutaneous vertebroplasty. Radiology 2006; 238(1): 211-220.

14. Syed MI, Jan S, Patel NA, et al. Fatal fat embolism after vertebroplasty: Identification of the high-risk patient. AJNR Am J Neuroradiol 2006; 27: 343-345.

15. Kauffman TJ, Trout AT, Kallmes DF. The effects of cement volume on clinical outcome of percutaneous vertebroplasty. AJNR Am J Neuroradiol 2006; 27: 1933-1937.

16. Edidin AA, Ong KL, Lau E, et al. Mortality risk for operated and non operated vertebral fracture patients in the medicare population. J BoneMiner Res 2011; 26(7): 1617-1626. 